\title{
Human Five Senses via Art
}

\author{
Abraham Tamir* \\ Chemical Engineering Department, Emeritus Professor of Ben-Gurion University of the Negev, Egypt
}

Submission: November 14, 2017; Published: December 04, 2017

*Corresponding author: Abraham Tamir, Chemical Engineering Department, Emeritus Professor of Ben-Gurion University of the Negev, Israel, Egypt, Email: atamir4@012.net.il

Mini Review
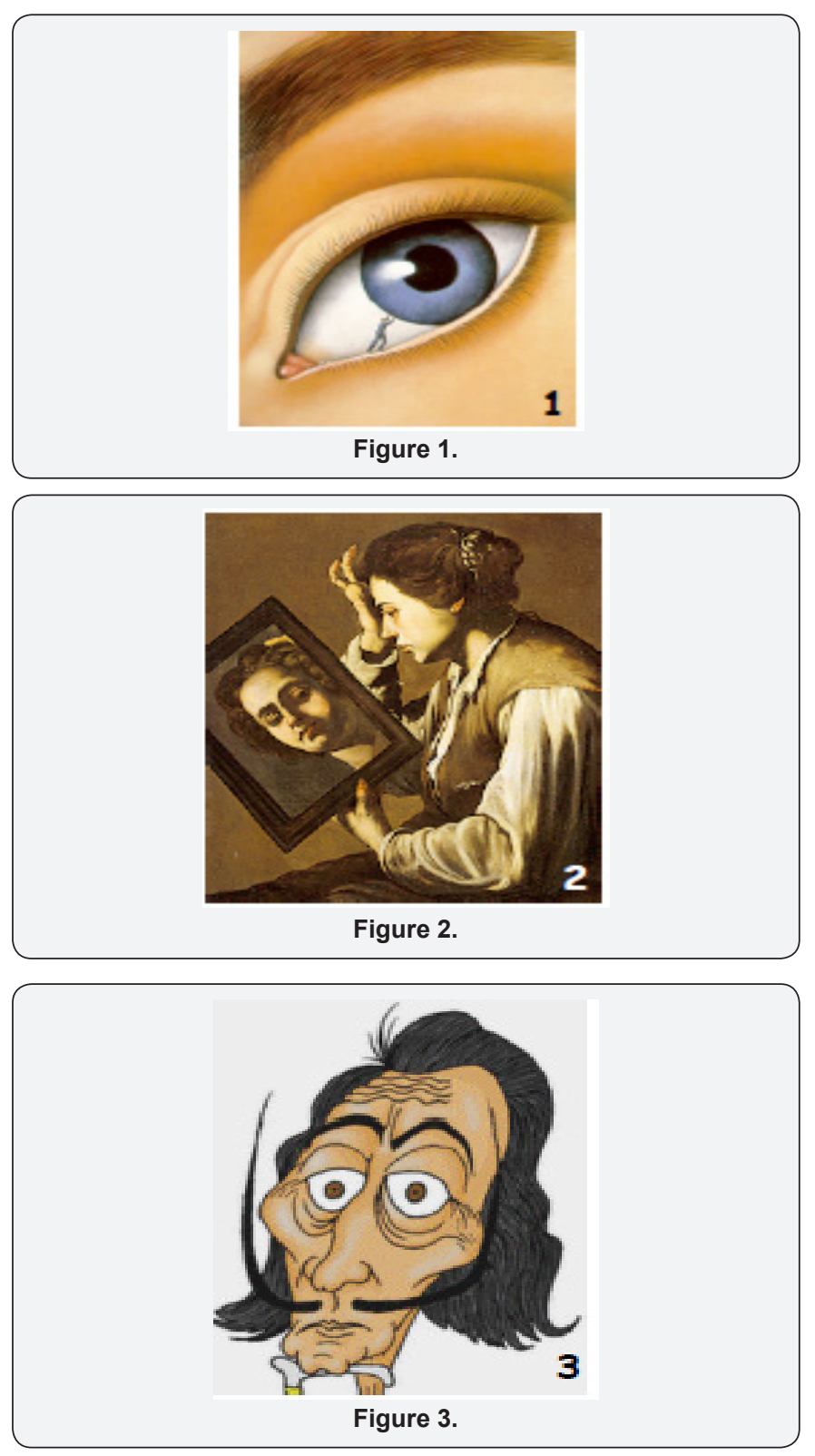

We experience the world around us by our five main senses, namely vision, hearing, touch, taste, and smell that are demonstrated in the following by artworks. Senses feed our brain with bits of information that help us to build a picture of the complicated world around us. Scientifically a sense is defined as a means of receiving information from the environment or from the body and converting it to an electrical - chemical code that is processed by the brain. The sense of vision demonstrated by Figures 1-3 is the most important one that enables us to see the world around us. It is defined as the perception of an object by the eye or the process, power or function of seeing. In the process of seeing light stimuli received by the eye are interpreted by the brain and constructed into a representation of the position, shape, brightness, and usually colour of objects in space.

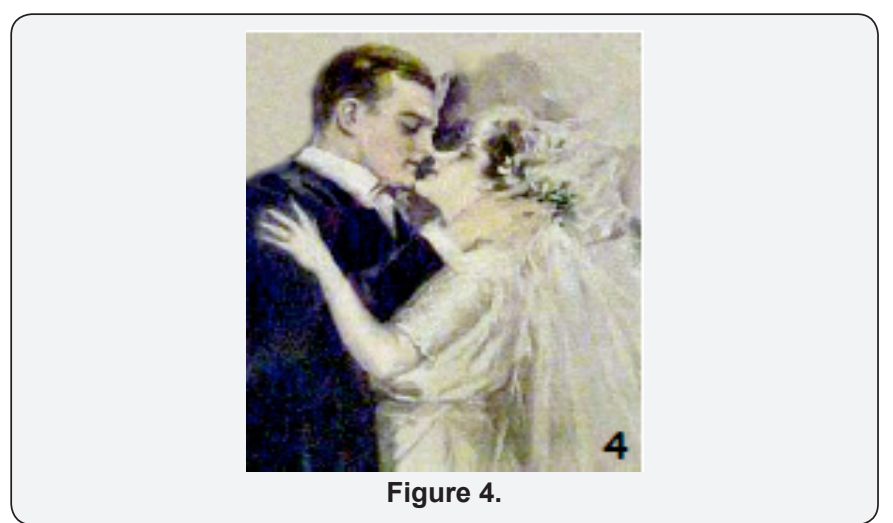

It enables also to distinguish between near and far objects, measure distances and create in the brain a three dimensional space. In Figure 1 painted by the Polish artist Rafael Olbinski we notice a small propelling of the middle of the eye pupil. In Figure 2 by the artist Spaniard Juan we see the reflectance mirror where in Figure 3 we observe a self-portrait of Salvador Dali that strongly emphasized the organ of vision. The sense of touch is demonstrated in Figures 4 \& 5 where the last one is by Rene Magritte. This sense is the sensitivity to a stimuli originating outside the body. It is interesting to emphasize 


\section{Global Journal of Addiction \& Rehabilitation Medicine}

this relationship from the thermodynamic point of view as an interaction between a "system", the skin, and its "surrounding", everything else.
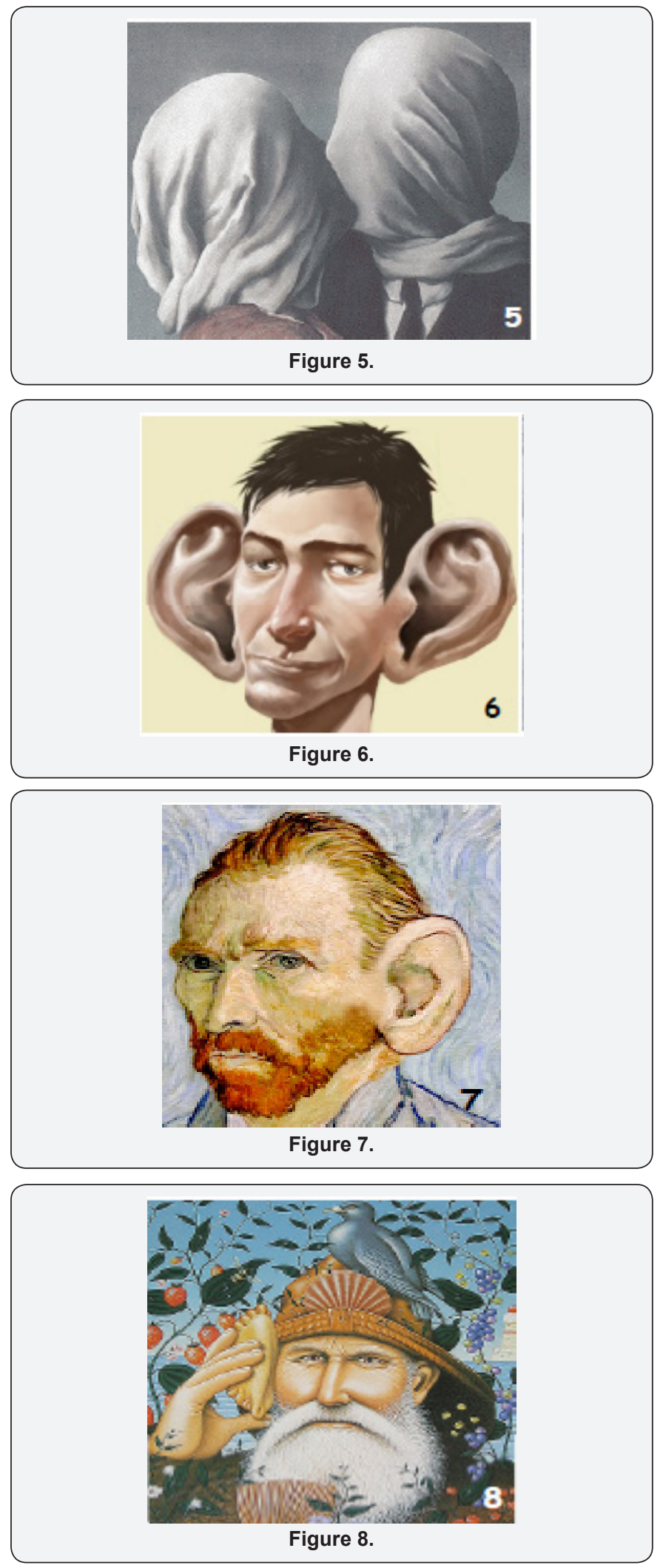

The next sense is the sense of hearing, which is the sense by which sound is perceived. This sense is the basis of interpersonal communication and the ability to capture sounds - sound waves - from the external environment with the ears. Figures $6 \& 7$ of the artist of the Dutch artist Van Gogh and Figure 8 of Games Marsh demonstrate the external ear, its auricle, which is the visible part of the ear that constitutes the first element with it the sound creates an interaction. The next sense is the sense of taste defined as the ability of distinguishing sweet, sour, bitter, and salty properties in the mouth that is created by receptors on the tongue. There are five tastes: sweet, salty, sour, bitter and umami that is a Japanese word for the taste imparted by glutamate in foods.

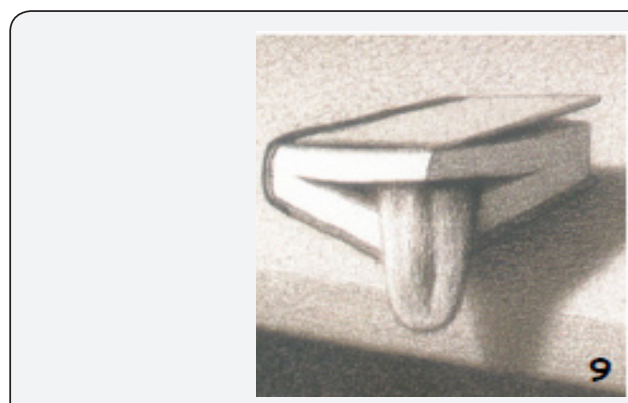

Figure 9.
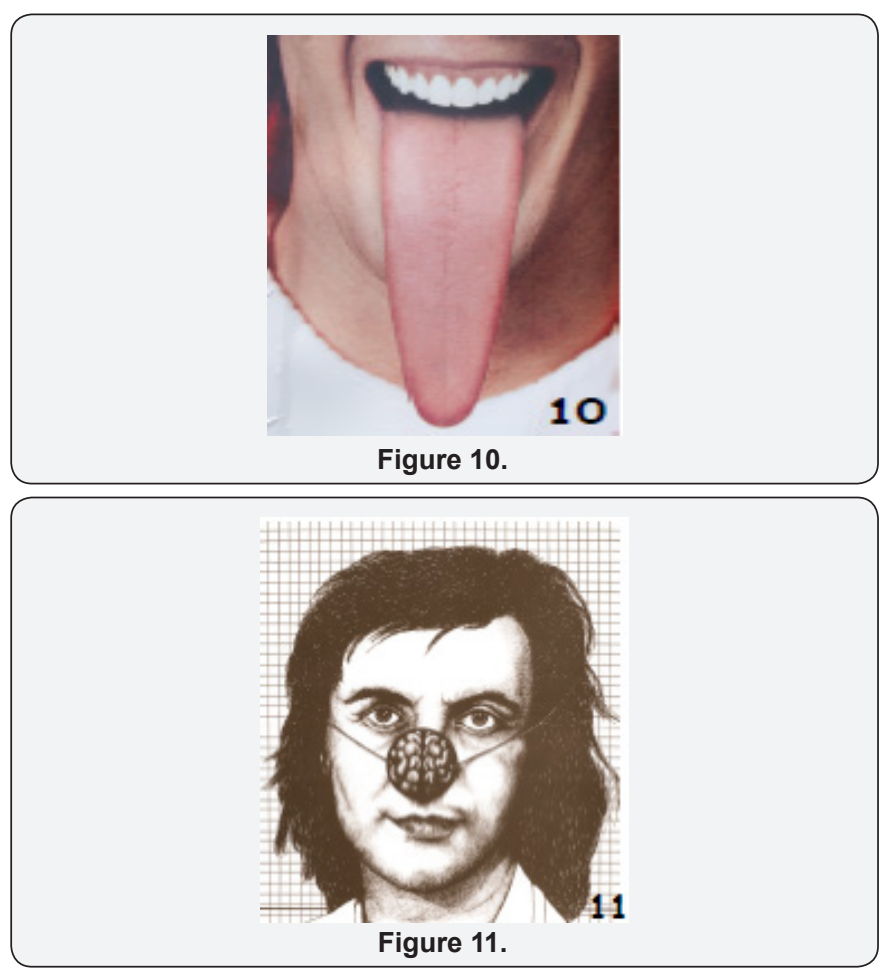

Figure 9 by the German artist Quint Buchholz and Figure 10 describe the sense of taste that allows distinguishing between tasty and delicious when the tongue is the primary organ of taste. This is emphasized in Figure 11 by Magritte due to the additional tongue. And finally the sense of smell that is the ability to distinguish volatile chemicals in air and odours. It is depicted in Figure 12 of Czech Oliver Solga and Figure 13 of the surrealist René Magritte where the picture of Solga emphasizes the fact that the smell eventually absorbed in the brain. Figure 14 is the image of Mona Lisa in which it is 


\section{Global Journal of Addiction \& Rehabilitation Medicine}

possible to distinguish the senses of vision, smell and touch where in Figures 11 \& 15 of Einstein all five senses can be observed.
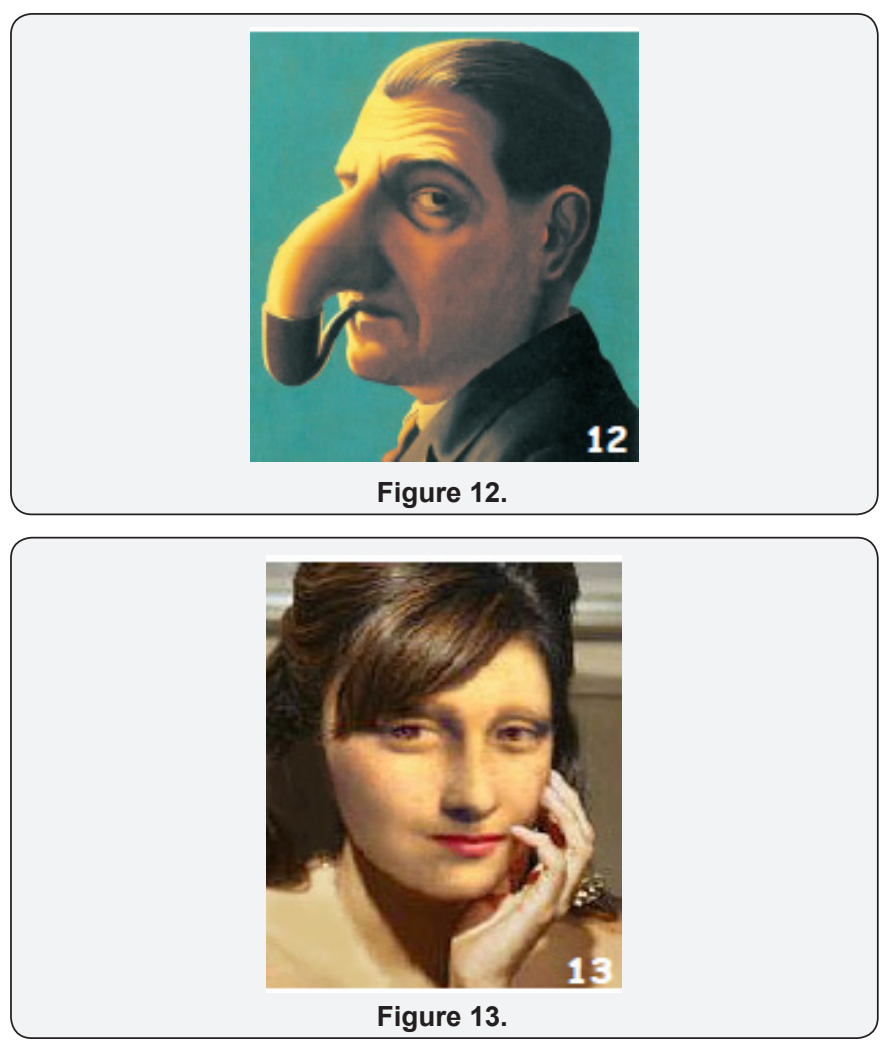

Figure 13.
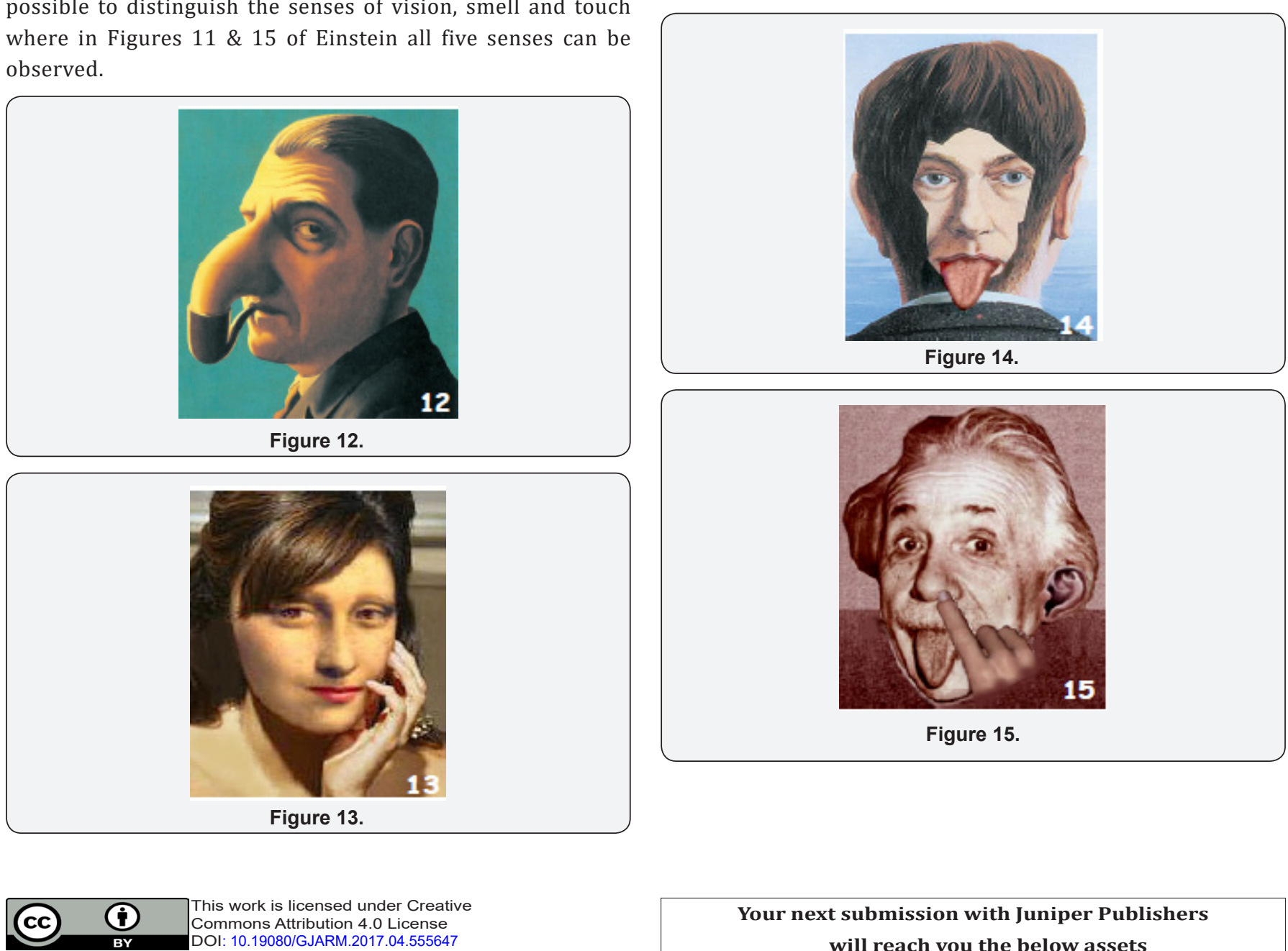